\title{
Utilizing Change Theory to Implement a Quality Improvement, Evidence- based Fall Prevention Model in Long-term Care
}

\author{
Cassandra Warner Frieson ${ }^{1 *}$, Dorothy Gargis Foote ${ }^{2}$, Karen H. Frith ${ }^{2}$ and John H. Wagner III \\ ${ }^{1}$ Long Term Care Physician Services of Alabama, Inc., 401 Arnold Street, Cullman, AL 35056, USA \\ ${ }^{2}$ The University of Alabama in Huntsville, Huntsville, AL, USA
}

\begin{abstract}
In order to maintain safety and increase the quality of life for Long Term Care (LTC) residents, fall prevention is a primary concern. Although there is conflicting evidence regarding the effectiveness of fall prevention strategies in LTC, there are evidence-based strategies that can be implemented to decrease fall rates. In order for these strategies to be effective, a behavioral change must occur from an organizational standpoint that includes key stakeholders such as administration, staff, residents, and their families. The authors sought to test the hypothesis, if a Change Theory Fall Model (CTFM) was implemented at the LTC facility, then behavioral changes will occur among key stakeholders to decrease fall rates in residents. The CTFM is an evidence-based fall prevention model developed by an Advanced Practice Nurse (APN) that incorporated Kotter and Cohen's Change Theory (2002). The CTFM was tested for its effectiveness in a non-profit, 119-bed nursing home, located in northern Alabama during January through December, 2011. Fall outcome measures for 2010 were compared with 2011. Although results did not reveal a decrease in fall rates for the specified time period, stakeholders did learn about Evidence Based Practice (EBP). Factors influencing fall rate outcomes included a high staff turn-over rate. Evaluation of the CTFM remains ongoing, and reeducation and data collection remain critical elements for success.
\end{abstract}

Keywords: Change theory; Geriatrics; Long-term care facilities; Nursing homes; Fall rates; Fall prevention strategies; Evidence-based strategies

\section{Introduction}

Approximately 1,800 fatal falls occur each year among United States Long-Term Care (LTC) residents. In addition, the total annual cost of falls among adults aged 65 and older, including fall-related mortalities, in the year 2000 was over $\$ 19$ billion and is estimated to increase to over $\$ 54.9$ billion by 2020 [1]. The greatest costs among older Americans are incurred upon hospitalization $(\$ 22,260)$; however, costs for treatment in emergency rooms and outpatient settings can be as high as $\$ 5,040$ [2]. According to the Centers for Disease Control and Prevention, $75 \%$ of residents aged 65 years and older who reside in LTC facilities fall each year. Many residents have recurrent falls averaging 2.6 falls per person per year, and many are injurious falls that can lead to the development of fractures and even death [1].

Multi-factorial evidence-based factors to consider in fall prevention education in LTC facilities include medication-induced side-effects, age-related physical and cognitive impairment, documentation policies and procedures, nurse staffing issues, and the professional's knowledge-base and educational background [3]. Although there is conflicting evidence of the effectiveness of multi-factorial interventions to decrease fall rates, results were favorable when interventions were provided from an interdisciplinary team approach [4]. Despite the interdisciplinary strategies supported by the evidence to decrease fall rates in residents [5-8], a behavioral change must occur from an organizational-wide standpoint and involve key stakeholders [9]. The authors sought to test the hypothesis, if a Change Theory Fall Model (CTFM) was implemented at the LTC facility, then behavioral changes will occur among key stakeholders to decrease fall rates in residents.

\section{Kotter and cohen's change theory}

Kotter and Cohen [10] developed a Model of Change that was presented in the book entitled The Heart of Change. The authors interviewed hundreds of individuals and businesses to receive real life stories from managers and companies on how they changed their organization. The authors gleaned from these stories how change functions, and they presented a model that served as a guide for organizations such as those seeking large-scale effective change. The primary message emphasized was that when an organization is attempting change, such as the development of new strategies, change is more likely to be effective when people are shown a truth that empowers individual feelings rather than given an analysis that alters ones thinking [10]. The authors stated that initiating change is complex, but that successful change happens when eight steps are taken to guide the change. These steps included the need to (a) create a feeling of urgency, (b) build the team to guide the change, (c) create a clear vision and realistic strategies, (d) communicate the vision and strategies with persuasive and emotional messages from real life situations, (e) empower behavioral changes and remove noted barriers, (f) create successes on a short-term basis, (g) continue to be persistent in promoting change, and $(\mathrm{h})$ nourish the new culture and behavior for ongoing growth. The authors stated that the primary challenge of all these steps is to promote behavioral change in the people [10].

\section{The CTFM}

The Advanced Practice Nurse (APN) developed a Change Theory Fall Model (CTFM) that integrated principles of Kotter and Cohen's Change Model (2002) with the most relevant synthesis of research evidence on fall prevention and management in LTC. The CTFM incorporated best practice fall interventions from 25 systematic reviews of randomized control trials (RCTs) and nonrandomized trials; clinical practice guidelines; single RCTs and nonrandomized

*Corresponding author: Cassandra Warner Frieson, DNP, CRNP, Long Term Care Physician Services of Alabama, Inc., 401 Arnold Street, Cullman, AL 35056, USA, E-mail: cwfrieson@charter.net

Received June 26, 2012; Accepted July 25, 2012; Published July 27, 2012

Citation: Frieson CW, Foote DG, Frith KH, Wagner III JH (2012) Utilizing Change Theory to Implement a Quality Improvement, Evidence-based Fall Prevention Model in Long-term Care. J Gerontol Geriat Res S1:001. doi:10.4172/2167-7182. S1-001

Copyright: (c 2012 Frieson CW, et al. This is an open-access article distributed under the terms of the Creative Commons Attribution License, which permits unrestricted use, distribution, and reproduction in any medium, provided the original author and source are credited. 
trials; and systematic reviews of co relational and observational studies. The CTFM served as a guide to clinical practice to promote evidencebased behavioral changes among key stake holders to reduce fall rates in LTC facilities; and the CTFM served as a Quality Improvement (QI) initiative with the aim to decrease a fall in each resident.

\section{Methods}

The CTFM was implemented at a 119-bed nursing home owned and operated by a nonprofit organization in northern Alabama. Nurse staffing consisted primarily of LPNs and CNAs under the supervision of RN Supervisors and Charge Nurses. The facility was comprised of $82 \%$ long-term and $18 \%$ short-term residents. The dates of implementation of the CTFM were from January through December, 2011.

Pre-CTFM behaviors exhibited by staff to promote resident safety consisted of standard fall prevention strategies. These strategies included the education of residents regarding the use of the call light for staff assistance prior to attempting to ambulate or get out of bed; the education of residents regarding the use of assistive aids and devices including glasses, hearing aids, walkers, canes, and/or wheelchairs; the implementation of bowel and bladder programs; the prevention of room clutter; the wearing of non-slid shoes; and the avoidance of ambulating on slippery surfaces. In compliance with federal guidelines in LTC, the RN Supervisor completed a Minimum Data Set (MDS) which is a comprehensive assessment of the functional capabilities of each resident. The MDS was completed upon admission, when a resident experienced a fall, and per MDS guidelines. Based on the selected responses of the MDS, a Resident Assessment Protocol (RAP) Summary was triggered that identified a resident's problem area for falls [11].

Although pharmacy medication review and consultation [8] was an integral component of the facility's fall prevention plan, there were other behaviors exemplified that warranted the use of the CTFM to promote effective change. Strategic areas found in need of a change included the use of the Fall Risk Assessment Form, resident group exercise program, vitamin D screening and supplementation, and fall educational meetings. The Fall Risk Assessment Form assessed the functional status of each resident utilizing eight parameters to identify a resident at high risk for falls [12]. The resident group exercise program consisted of a 30 minute twice-a-week instructor-led group activity that primarily focused on upper and lower extremity muscle strengthening. Laboratory work-up of the residents, although performed on a routine basis, did not include routine screening of residents for vitamin $\mathrm{D}$ deficiency; and fall educational meetings, although held on a daily basis on week days to include the administrator, DON, and staff from multiple disciplines, lacked a comprehensive interdisciplinary team that was guided by an APN. These pre-CTFM behaviors were targeted to promote a change toward evidence-based practice (EBP) to decrease fall rates.

After approval from the University Institutional Review Board (IRB) and corporate administration, the APN served as liaison and met with corporate administration, nursing, and ancillary staff to implement the CTFM. A feeling of urgency was created regarding the need to promote EBP such as the use of the Morse Fall Scale [13], Tai Chi Exercises [5], and vitamin D supplementation [6,7]. Attendees were told stories of real-life situations of residents who had exhibited a decreased quality of life due to a sustained fall that resulted in a fracture or head trauma. Emphasis was placed on how EBP can really make a difference in these and other residents by preventing further injuries and even death. A clear vision was established with a focus on resident safety as reflected in the facility's vision, mission, and goals.

An interdisciplinary fall response team was organized that included the APN, physician, administrator, Director of Nursing (DON), QI nurses, Registered Nurse (RN) supervisors, Licensed Practical Nurses (LPNs), restorative nurses, and Certified Nursing Assistants (CNAs). Ancillary staff included the pharmacist, therapy directors (Physical, Occupational, and Speech), activity director, dietician, social worker, and maintenance and housekeeping directors. In addition to the previously established practice of pharmacy medication review and consultation [7], the team, as well as the residents and their families, were educated on other evidence-based strategies to decrease fall rates and injurious falls (Figure 1).

The APN developed and implemented a Fall Management Protocol that included time-lines for fall management. Within 24 hours of a resident's admission, the RN supervisor or Charge Nurse obtained a fall history, performed a MFS assessment, notified the APN and/ or physician of the resident's admission fall status, and completed fall documentation. The QI nurse updated the interdisciplinary team regarding the facility's current fall rate statistics and fall related injuries. The APN , with the assistance of the team, established individualized plans of care for high risk residents to include such interventions as low bed and mat placement; the use of monitoring and alarm systems (e.g, bed and chair alarms); and the evaluation of the resident's medication profile for medication adjustments and/or discontinuations. After a fall event for an established or long-term resident, the RN supervisor or Charge Nurse performed the initial 24-hour fall interventions, assessed the resident for injuries, and notified the fall coordinators (e.g., DON and QI Nurses), the APN and/or the physician. Within the first seven days of the initial fall for admitted and established

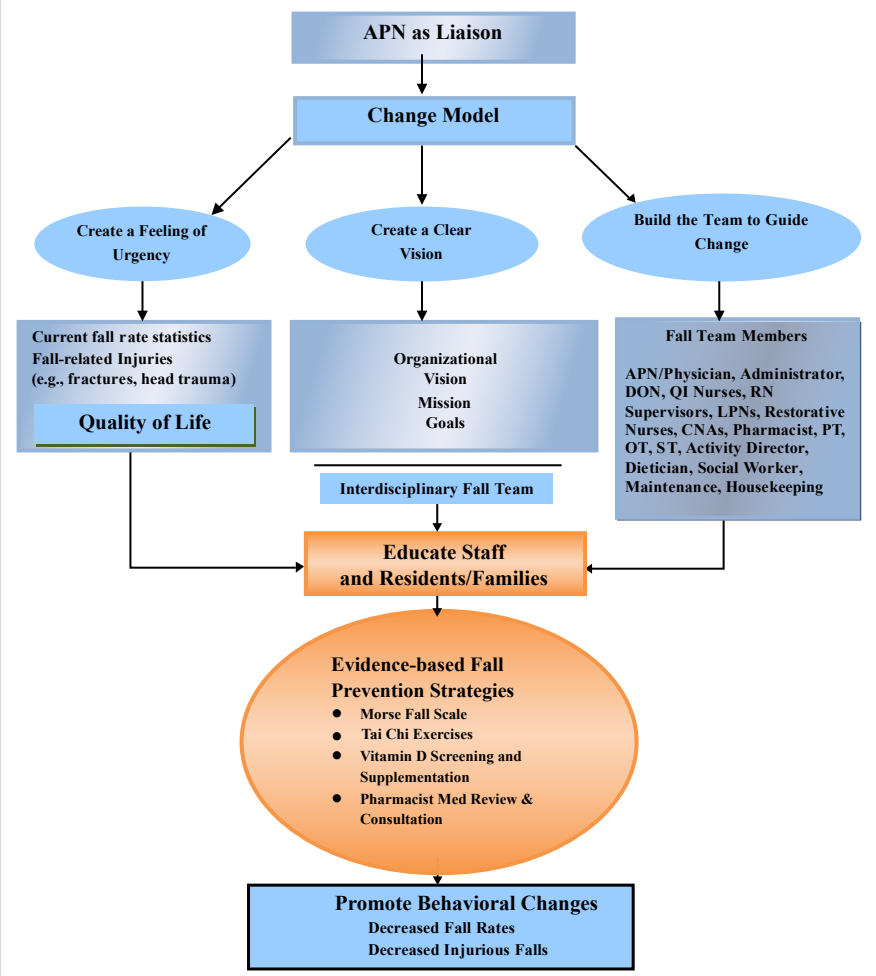

Figure 1: Steps to Prevent a Fall in the LTC Resident through Behavioral Modification Utilizing Principles of Kotter and Cohen's Change Model. 
Citation: Frieson CW, Foote DG, Frith KH, Wagner III JH (2012) Utilizing Change Theory to Implement a Quality Improvement, Evidence-based Fall Prevention Model in Long-term Care. J Gerontol Geriat Res S1:001. doi:10.4172/2167-7182.S1-001

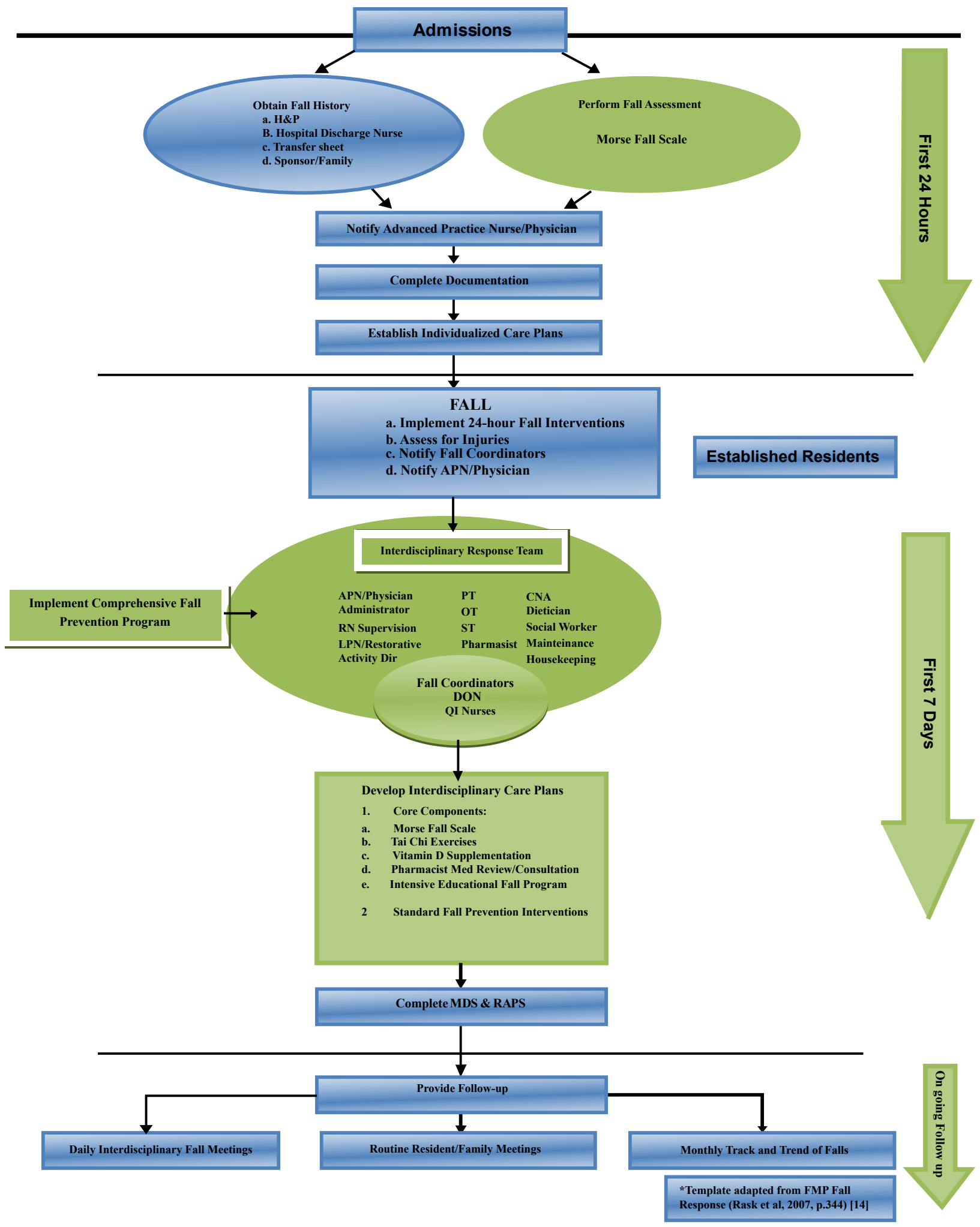

Abbreviations: CTFM; Change Theory Fall Model; H \& P: History \& Physical exam; APN: Advanced Practice Nurse; DON: Director Of Nursing; QI Nurses: Quality Improvement Nurses; RN: Registered Nurse; LPN: Licensed Practical Nurse; CNAs: Certified Nurse Assistants; PT: Physical Therapist; OT: Occupational Therapist; ST: Speech Therapist; Activity Dir.: Activity Director; Pharmacist Med Review: Pharmacist Medication Review; MDS: Minimum Data Set; RAPS: Resident Assessment Protocol Summary; FMP: Fall Management Program:

Figure 2: Evidence-based Fall Management Protocol for Long-term Care. 
residents, the coordinators activated the interdisciplinary response team to implement core components. With the guidance of the APN, a comprehensive, interdisciplinary fall prevention program was established [14], thus, granting the APN the opportunity to provide repeated emphasis on the components that need to be implemented by team members and staff. Standard fall interventions were on-going and included the completion of the MDS and RAPS. Follow-up for each fall resident included daily interdisciplinary fall meetings, routine resident/family meetings, and monthly involvement of the QI nurse to track and trend falls (Figure 2).

\section{Fall definitions}

According to the Centers for Medicare and Medicaid Services (CMS) Minimum Data Set (MDS) 3.0 Resident Assessment Manual, a fall occurrence is defined as an unintentional event that results when a resident has a change in position that has caused the resident to land on the floor, next surface beneath the resident (e.g floor mat, bed, chair) (witnessed), or a report of a resident who has landed on the floor (unwitnessed). A fall occurs when a resident has rolled off a low bed or mattress and landed on the floor. A slip, trip, or stumble that results in a position change is still categorized as a fall event; even if the fall is prevented due to the resident's regaining balance or being intercepted by another person to prevent the fall [11].

\section{Sample demographics and selection}

Fall prevention and management strategies were targeted toward all residents of the facility. Residents were categorized into two groups: those who were short-term, 21-day rehabilitation residents and those who were long-term residents. For the purpose of this study, fall data were collected and analyzed on long-term residents because their inclusion allowed adequate follow-up for the evaluation of fall rates. The participants were established facility dwellers with a length of residency that varied from approximately 1 to 13 years. The residents' average age was greater than 75 years, and they were impaired or low functioning residents with some degree of cognitive decline. The majority of residents who fell were white females with an average age of 83.1 years (variation in ages from 66 to 94 years) with a high school or $8^{\text {th }}$ grade education. Two residents had some college and a graduate degree, respectively. Short-term rehabilitation residents were excluded from data collection.

\section{Data collection and analysis}

The APN consulted with the QI nurse and reviewed the Incident Analysis Log to collect fall data for each quarter for the year 2011. For confidentiality, each resident was identified by a resident number. The categories listed on the log included the (a) date and time of incident, (b) type of incident (e.g., falls with no head injury or found on the floor), (c) reportable incident (eg. involvement of the State Health Department), (d) type of injury (eg. none apparent or fracture), (e) location of occurrence (eg. resident's room or bathroom), and (f) the disposition type (eg. in-house treatment or hospitalization). Demographic data and fall circumstances (eg. self transfer from bed to wheelchair) were obtained from chart reviews.

\section{Outcome measures}

The primary outcome measures included the adoption of the CTFM. Secondary outcome measures included the post-CTFM evidence-based behaviors and resident fall rates. Fall rate, or rate ratio was defined as the actual number of reported person falls per year; fallers were defined as the number of persons who experienced a fall $[4,13]$. According to the CMS Minimum Data Set 3.0 Version for nursing facilities [11], fall categories can be grouped into major and non-major injurious falls. Major injurious falls included fractures, joint dislocations, closed head injuries (with altered mental status), and subdural hematomas. Non-major injurious falls included skin tears, abrasions, lacerations, discolorations/bruises, and hematomas. A resident fall without reported injury was defined as a non-injurious fall (Tables 1 and 2).

\section{Post-CTFM evidence-based core components}

Annually, a typical 100-bed nursing home has a reported 100 to 200 fall incidence rate [1]. Although no one scale is ideal in predicting a fall in residents, choosing an instrument for clinical practice should take into account the sensitivity and specificity of the instrument $[15,16]$. The Morse Fall Scale (MFS) is a valid tool used to detect the presence of falls (sensitivity of 72\%) and identify fall absence (specificity of $51 \%)$. The MFS is a widely used fall risk assessment scale that has been shown in research studies to identify a resident at increased risk for a fall. The MFS consists of six variables of risk factors. The scale variables include (a) fall history, (b) secondary diagnosis, (c) ambulatory aid, (d) intravenous therapy/heparin lock usage, (e) gait, and (f) mental status. Inter rater reliability estimations, $r=.96$ was established by the rating of six patients by 21 nurses using the first five variables. Based on the selected responses, a total score is obtained to identify the resident's risk for a fall $[13,17,18]$. It is important to note that the MFS did not capture fall risk due to wheelchair use; and research studies have shown a significant correlation between these two variables $(\mathrm{r}=0.406, \mathrm{p}=$ 0.001 ) [19]. Therefore, high risk residents are placed at an even greater fall risk if they are wheelchair bound.

Tai Chi exercises are supported by the evidence to decease fall rates in the elderly [20]. Tai Chi exercises are gentle, slow, continuous movements performed with a very high stance suitable for the elderly to improve balance, strength, and postural stability. Current evidence supports the use of Tai Chi to decrease fall rate and risk and the fear of falling; and Tai Chi has been shown to be effective in the physically inactive elderly aged 70 years or greater to improve functional balance and physical performance $[5,20]$.

Studies have shown that a majority of residents (89\%) who reside

\begin{tabular}{|c|c|c|c|c|c|}
\hline Fall Indicators & $\begin{array}{c}\text { Quarter } \\
1\end{array}$ & $\begin{array}{c}\text { Quarter } \\
2\end{array}$ & $\begin{array}{c}\text { Quarter } \\
3\end{array}$ & $\begin{array}{c}\text { Quarter } \\
4\end{array}$ & Total \\
\hline Number of Falls & 21 & 39 & 30 & 13 & 103 \\
\hline Number of Fallers & 15 & 20 & 17 & 9 & 61 \\
\hline Non-injurious falls $^{1}$ & 15 & 31 & 18 & 8 & 72 \\
\hline $\begin{array}{l}\text { Injurious falls (Major) }{ }^{1} \\
\text { a.Fractures (Fxs) } \\
\text { b. Joint Dislocations } \\
\text { c. Closed Head Injuries } \\
\text { (altered mental status) } \\
\text { d. subdural Hematoma } \\
\text { e. Other } \\
\text { Injurious falls (Non-Major) }{ }^{1} \\
\text { a. Skin tears } \\
\text { b. Abrasions } \\
\text { c. Lacerations } \\
\text { d. Discolorations/Bruises } \\
\text { e. Hematomas } \\
\text { f. Other }\end{array}$ & $\begin{array}{c}2 \text { Hip Fxs } \\
0 \\
0 \\
\\
0 \\
0\end{array}$ & $\begin{array}{l}3 \\
2 \\
0 \\
1 \\
2 \\
0\end{array}$ & $\begin{array}{l}0 \\
0 \\
0 \\
0 \\
0\end{array}$ & $\begin{array}{l}0 \\
0 \\
0 \\
0 \\
0\end{array}$ & $\begin{array}{c}2 \text { Hip Fxs } \\
0 \\
0 \\
\\
0 \\
0\end{array}$ \\
\hline Total Census & 63 & 60 & 64 & 60 & $60-64$ \\
\hline
\end{tabular}

${ }^{1}$ Fall indicators according to the CMS Minimum Data Set (MDS) 3.0 Version in nursing homes

Table 1: Number of Falls, Fallers, and Resultant Injuries for LTC Residents per Quarter for the Year 2010. 


\begin{tabular}{|c|c|c|c|c|c|}
\hline Fall Indicators & $\begin{array}{c}\text { Quarter } \\
1\end{array}$ & $\begin{array}{c}\text { Quarter } \\
2\end{array}$ & $\begin{array}{c}\text { Quarter } \\
3\end{array}$ & $\begin{array}{c}\text { Quarter } \\
4\end{array}$ & Total \\
\hline Number of Falls & $34(21)$ & 33 & 31 & 31 & 129 \\
\hline Number of Fallers & $20(" 15)$ & 18 & 15 & 22 & 75 \\
\hline Non-injurious falls ${ }^{1}$ & $26(17)$ & 19 & 21 & 24 & 90 \\
\hline $\begin{array}{l}\text { Injurious falls (Major) } \\
\text { a.Fractures (Fxs) } \\
\text { b. Joint Dislocations } \\
\text { c. Closed Head Injuries } \\
\text { (altered consciousness) } \\
\text { d. subdural Hematoma } \\
\text { e.Other } \\
\text { Injurious falls (Non- } \\
\text { Major) } \\
\text { a. Skin tears } \\
\text { b. Abrasions } \\
\text { c. Lacerations } \\
\text { d. Discolorations/Bruises } \\
\text { e. Hematomas } \\
\text { f. Other }\end{array}$ & $\begin{array}{c}1 \text { Elbow Fxs } \\
0 \\
0 \\
0 \\
0 \\
0 \\
0 \\
0 \\
2 \\
3 \\
1 \text { Puncture } \\
\text { Wound }\end{array}$ & $\begin{array}{l}6 \\
1 \\
1 \\
4 \\
2 \\
0\end{array}$ & $\begin{array}{l}4 \\
0 \\
1 \\
6 \\
1 \\
0\end{array}$ & $\begin{array}{c}1 \text { Femur Fxs } \\
0 \\
0 \\
0 \\
0 \\
1 \\
2 \\
1 \\
3 \\
1 \\
1 \text { Tissue } \\
\text { Edema( } \\
\text { Localized) }\end{array}$ & $\begin{array}{c}11 \\
8 \\
3 \\
15 \\
7 \\
2\end{array}$ \\
\hline Total Census & 64 & 63 & 63 & 64 & $63-64$ \\
\hline
\end{tabular}

*Number of falls utilizing the Morse Fall Scale

${ }^{1}$ Fall indications according to the CMS Minimum Data Set (MDS) 3.0 Version in nursing homes

Table 2: Number of Falls, Fallers, and Resultant Injuries for LTC Residents Per Quarter for the Year 2011.

in LTC facilities are vitamin D deficient due to decreased exposure to sunlight, decreased dietary intake, and multiple co-morbidities [21]. Long-term vitamin D supplementation of $800 \mathrm{IU}$ per day in residents whose levels are low $(<25 \mathrm{ng} / \mathrm{ml}$ total serum 25 -hydroxyvitamin $\mathrm{D}$ level) decreases fall rates, fallers, and injurious falls resulting in fractures [6,7].

According to the evidence, the implementation of an effective fall management program should be comprehensive and include leadership support and guidance from an APN. Core components should include a fall coordinator, an interdisciplinary team, and an intensive training and educational program to prevent further increase in fall rates [14].

\section{Results}

Corporate administration approved and adopted the CTFM for facility-wide use. Post-CTFM evidence-based behaviors included the approval and computerized use of the MFS; the use of Tai Chi exercises, vitamin D screening and supplementation; and an APN guided fall management program. There was no significant reduction in the facility's fall rates.

The previously used Fall Risk Assessment Form was replaced with the MFS. The APN conducted training sessions for the interdisciplinary team members on a weekly basis utilizing MFS videos with round-table discussions. The APN gave instructional guidance and encouragement to the team; consistent use of the MFS was shown. Residents were assessed or reassessed utilizing the MFS upon admission to the facility, significant change in overall condition, change in medications (eg. psychotropic, cardiovascular, and diuretics), and/or after a fall event. The RN Supervisor or Charge Nurse completed the MFS and obtained an overall score to determine the resident's fall risk. The resident was designated at high risk if the MFS score was greater than 45 (see Janice Morse Preventing Patient Falls $2^{\text {nd }}$ edition for calibration of scores). The ultimate goal of each resident was to decrease the resident's MFS score and reduce fall rates [13]. In consultation with the QI nurse, for the year 2011, staff utilization of the MFS has led to a more prompt identification of residents at high-risk for falls. The MFS was described as more "user friendly" and included an interactional assessment, similar to the previously used assessment tool, that allowed the examiner to assess each resident's functional abilities through observation of the resident's gait impairment and balance. Permission for MFS use was granted by Janice M. Morse, PhD, FAAN.

Tai Chi exercises replaced the routine resident group exercise activities. The APN gave instructional guidance after consultation with the Physical Therapist, Activity Director, and interdisciplinary team, who reviewed the unique concepts of the Tai Chi principles as outlined in an in-service manual [22]. With encouragement from the APN, the team and residents were receptive and adhered to Tai Chi instruction. The team performed practice sessions to familiarize themselves with Tai Chi techniques. Resident group participation included approximately 25 residents per session. Family participation was encouraged as well with limited participation received. The residents experienced a 30-minute twice-weekly Tai Chi work-out on Mondays and Thursdays that was instructor-lead utilizing DVD videos and selected members of the interdisciplinary team to include the Activity Director, Activity Assistants, Restorative Nurses, and Physical Therapists. Due to the fact that a majority (91\%) of the residents participating in the study was primarily non-ambulatory, Tai Chi principles were incorporated into the wheelchair-bound residents' exercise regimen and remained the central focus of the movement exercise [23]. The APN held faceto-face interviews with residents to inquire about their satisfaction with the Tai Chi program. The benefits reported included overall satisfaction with the Tai Chi program as opposed to the previous group exercise program. The majority of residents (both ambulatory and non-ambulatory) reported positive effects of Tai Chi exercises on their health that included feeling stronger (eg. improved muscle strength and endurance), experiencing less pain (eg. joint pain/stiffness from arthritis), and feeling more relaxed (eg. improved overall psychological well-being). Residents with severe cognitive impairment, critically ill, and/or in a frail state were excluded from participation in Tai Chi exercises.

The phlebotomist incorporated Vitamin D screening as a component of the standard admission and routine laboratory workup for residents. Vitamin D levels were obtained on all residents upon admission, quarterly, and on an as needed basis. Approximately $90 \%$ of the nursing home residents who participated in the study were vitamin $\mathrm{D}$ deficient and were supplemented accordingly with levels reaching therapeutic range.

A Fall Management Protocol was developed by the APN as part of a comprehensive fall educational program to include the education of staff, resident, and their families (Figure 2). Staff compliance was exemplified with adherence to the protocol. Residents and their families were educated on fall prevention strategies. A resident who was identified as high-risk received the placement of a yellow-coded fall bracelets on the arm; and yellow-coded name tags replaced the standard white name tags on the resident's door. Also, clip-on alarms replaced the stringed alarms to alert staff of the resident's unsafe acts and facilitate resident compliance. As a result of such changes and educational efforts, an increase in awareness of a high-risk resident was displayed by the interdisciplinary team, nursing, and ancillary staff.

There was no significant reduction in the facility's fall rates. Evaluation of fall rates for the year 2010 was 62 residents. The annual number of falls, fallers, and non-injurious falls per year was $n=103, n$ $=61$, and $n=72$ respectively. The number of major injuries remained relatively low; two residents had hip fractures. There were residents who had multiple non-major injuries (eg. skin tears and bruises). Many 
residents who experienced falls had recurrent falls; many resident falls did not result in injuries (Table 1). Post-CTFM, the average quarterly census for the year 2011 remained relatively consistent at 64 residents. The number of falls, however, remained consistently high. The annual number of falls, fallers, and non-injurious falls per year was $n=129, n=$ 75 , and $\mathrm{n}=90$ respectively. The number of major injuries remained low and included a resident with an elbow fracture and another resident with a femur fracture; there were no hip fractures. There were multiple non-major injuries. The MFS was approved by the QI committee during the later part of the first quarter, and approximately $90 \%$ of residents received a MFS assessment for the year 2011 (Table 2).

\section{Conclusions}

Despite the successes in the planned evidence-based behavioral changes post-CTFM that involved key stakeholders, results did not reveal a significant decrease in fall rates. There were critical elements that might have contributed to this lack of change. Even though the staff-to-resident ratio remained consistent, the staff turn-over rate increased from $5.5 \%$ to $10 \%$ during the year 2011 . There was also a change in nursing administration during the later part of the fourth quarter; thus, the APN had to re-devote time to intensive fall training, reeducation, and data collection. The MFS was not utilized on all residents during the first quarter in 2011 due to time devoted to the approval process by the QI committees prior to scale use. The scale was utilized on all residents during the remaining three quarters. The MFS did not capture wheelchair-bound residents for increased fall risk. These individuals comprised a majority of LTC residents. The APN consulted with the QI nurse on a routine basis to track and trend fall risks for wheelchair-bound residents.

The frequency of Tai Chi exercises was performed twice-weekly; however, an increase in the exercises to three-times-weekly has been shown to be more beneficial in decreasing fall rates [19]. There was a need for consistent encouragement from the APN and team for increased participation in the Tai Chi program from the residents and their families due to low turn-out rates. Although pharmacy consultation was an established component of the fall prevention plan, and the medical team adhered to the majority of pharmacy recommendations, data on percent adherence were not obtained. Clinical knowledge of these results would have provided a better understanding of fall rate outcomes. Subsequently, the classification of a fall was a critical element that might have influenced outcomes; whereas the United States Department of Veterans Affairs does not consider an intercepted fall such as a slip, trip, or stumble to result in a fall [24], the CMS guidelines for nursing homes considered these circumstances as fall events.

\section{Implications for clinical practice}

There are fall prevention strategies supported by the evidence that can decrease fall rates in LTC facilities [5-8]. In order for these strategies to be implemented effectively, a behavioral change must occur among key stakeholders such as administration, staff, residents and their families [9]. The analysis of stakeholders is an essential component in laying the foundation for organizational change to promote effective QI initiatives and expected outcomes [9]. Although the fall outcomes did not improve, stakeholders were educated and changed behaviors that supported EBP; and educating key stakeholders on evidence-based care is essential to nursing practice [25]. From an organizational standpoint, the implementation of best-practices has led to no fall-related deficiencies by the Department of Health and Human Services' CMS for the year 2011 as compared to 2010. The facility was chosen as finalist for the 2011 Alabama Best Practices by the Alabama Nursing Home Association (ANHA) for exemplary performance in fall prevention and management in LTC. The complexity involved in behavioral change requires the cooperation and commitment from key stakeholders. Learning from the process is valuable to all, particularly when an emphasis on fall prevention instills confidence in quality of care.

\section{Acknowledgements}

I am thankful for the support of colleagues, graduate professors, and staff at The University of Alabama in Huntsville, Huntsville, AL. Doctor of Nursing Practice (DNP) Program, as well as corporate and facility administration, QI nurses, and nursing and ancillary staff at LTC Physician Services of Alabama, Inc, Cullman AL. Finally, I am thankful for God's strength and provision of insight that has guided me in completion of this manuscript and the support received by my co-authors husband, Tommy Frieson; children, Tomeka and Tamara; and friends.

\section{References}

1. Centers for Disease Control and Prevention. Falls in nursing homes (2012).

2. Finkelstein EA, Chen H, Miller TR, Corso PS, Stevens JA (2005) A comparison of the case-control and case-crossover designs for estimating medical costs of nonfatal fall-related injuries among older Americans. Med Care 43: 1087-1091.

3. Agency for Healthcare Research and Quality (AHRQ). Falls prevention literature review: multiple interventions and implementation strategies (2012).

4. Cameron ID, Murray GR, Gillespie LD, Robertson MC, Hill KD, et al. (2010) Interventions for preventing falls in older people in nursing care facilities and hospitals (Review). Cochrane Database of Systematic Reviews.

5. Choi JH, Moon JS, Song R (2005) Effects of Sun-style Tai Chi exercise on physical fitness and fall prevention in fall-prone older adults. J Adv Nurs 51: 150-157.

6. Broe KE, Chen TC, Weinberg J, Bischoff-Ferrari HA, Holick MF, et al. (2007) A higher dose of vitamin $D$ reduces the risk of falls in nursing home residents: a randomized, multiple-dose study. J Am Geriatr Soc 55: 234-239.

7. Law M, Withers H, Morris J, Anderson F (2006) Vitamin D Supplementation and the prevention of fractures and falls: results of a randomised trial in elderly people in residential accommodation. Age Ageing 35: 482-486.

8. Verrue CL, Petrovic M, Mehuys E, Remon JP, Vander Stichele R (2009) Pharmacists' interventions for optimization of medication use in nursing homes: a systematic review. Drugs Aging 26: 37-49.

9. Alvord B (2008, Jan 9) Stakeholder analysis-key to productivity initiatives.

10. Kotter JP, Cohen DS (2002) The heart of change: real-life stories of how people change their organizations. Harvard Business School Press, Boston.

11. Centers for Medicare \& Medicaid Services. Nursing Home Quality Initiatives: MDS 3.0 Training Materials (2011).

12. Briggs Healthcare. Fall Risk Assessment (1997)

13. Morse JM (2009) Preventing patient falls. (2nd eds), Springer Publishing Company, New York.

14. Rask K, Parmelee PA, Taylor JA, Green D, Brown H, et al. (2007) Implementation and evaluation of a nursing home fall management program. J Am Geriatr Soc 55: 342-349.

15. Chapman J, Bachand D, Hyrkas K (2011) Testing the sensitivity, specificity, and feasibility of four falls risk assessment tools in a clinical setting. $\mathrm{J}$ Nurs Manag 19: 133-142.

16. Kehinde JO (2009) Instruments for measuring fall risk in older adults living in long-term care facilities: an integrative review. J Gerontol Nurs 35: 46-55.

17. Morse JM (1996) Preventing patient falls. (1 ${ }^{\text {st }}$ eds), Sage Publications, Thousand Oaks, CA.

18. Morse JM, Morse RM, Tylko SJ (1989) Development of a scale to identify the fall-prone patient. Canadian Journal on Aging 8: 366-377.

19. Fonad E, Wahlin TB, Winblad B, Emami A, Sandmark H (2008) Falls and fal risk among nursing home residents. J Clin Nurs 17: 126-134.

20. Li F, Harmer P, Fisher KJ, McAuley E, Chaumeton N, et al. (2005) Tai Chi and fall reductions in older adults: a randomized controlled trial. J Gerontol A Bio Sci Med Sci 60:187-194. 
Citation: Frieson CW, Foote DG, Frith KH, Wagner III JH (2012) Utilizing Change Theory to Implement a Quality Improvement, Evidence-based Fall Prevention Model in Long-term Care. J Gerontol Geriat Res S1:001. doi:10.4172/2167-7182.S1-001

21. Schilling S (2012) Epidemic vitamin D deficiency among patients in an elderly care rehabilitation facility. Dtsch Arztebl Int 109: 33-38.

22. Horve-Willoughby C, Tuesca B, Cross Country Education Instant In-services (2006). Tai Chi for balance, fall risk reduction and rehabilitation.

23. Broda LC (2004) The Tai Chi and Chi Kung forum for health (UK).
24. United States Department of Veterans Affairs (2009). National Center for Patient Safety (NCPS) fall prevention and management.

25. Malloch K, Porter-O'Grady T (Eds.) (2010) Introduction to evidence-based practice in nursing and health care. ( $2^{\text {nd }}$ eds), Jones and Bartlett, Sudbury, MA 